\title{
A Mathematical Model of the Inflammatory Response to Pathogen Challenge
}

\author{
Lester Caudill ${ }^{1, *}$, Fiona Lynch $^{1}$ \\ ${ }^{1}$ Department of Mathematics \& Computer Science, University of Richmond, Richmond, VA 23173 \\ lcaudill@richmond.edu
}

The human body's immune response to bacterial challenge, even when successful in controlling the infection, can result in negative consequences for the host, including reduced functionality of associated tissues. We present and analyze a low-dimensional mathematical model of this immune response to pathogen invasion, incorporating the coordinated actions of active immune cells, and both pro- and anti-inflammatory cytokines. The model simulates both the positive (pathogen reduction) and negative (local tissue dysfunction) effects of the immune response, and includes the important role of immunologic memory in the process of a return to stasis. This differential equations-based model is sufficiently general to be applicable to a wide-range of human tissues and organs. 